\title{
Complementary use of Label-Free Real-Time Biosensors in Drug Discovery of Monoclonal Antibodies
}

\author{
Yasmina Noubia Abdiche \\ Pfizer, Inc \\ United States of America
}

\section{Introduction}

Biosensors measure the real-time reversible interactions between biologically-relevant molecules independent of labels. Typically, they harness an optical phenomenon to detect the binding of a solution molecule (analyte) to its immobilized partner (ligand) on a sensor and record the output as a "sensorgram", which tracks the binding signal in arbitrary units proportional to mass sensed at the surface of the biosensor as a function of time. These cutting-edge biophysical tools are revolutionizing our understanding of molecular-level processes because they reveal an entire binding profile between unlabeled reactants and dissect it into kinetic components from which an affinity can be deduced. By definition, the affinity or equilibrium dissociation constant $\left(K_{\mathrm{D}}\right.$, in units of molar concentration, $\left.\mathrm{M}\right)$ is the ratio of the kinetic rate constants $\left(k_{\mathrm{d}} / k_{\mathrm{a}}\right)$ where $k_{\mathrm{d}}$ is the dissociation rate constant in units of reciprocal seconds $(1 / \mathrm{s})$ and $k_{\mathrm{a}}$ is the association rate constant in units of $1 /$ Ms. Knowing the affinity of an interaction is important to drug discovery because it affects the dose at which a drug is efficacious; drugs that bind their targets tightly can be administered at lower levels than those that bind weakly and thus are less likely to exhibit undesirable side-effects and are more cost-effective. End-point analyses, like ELISA, are less informative and rely upon labeling the reactants and adsorbing one of them, which can spoil their native activity, give high non-specific background signals, and limit the scope of an assay. Biosensors are commonplace in industrial and academic research laboratories worldwide and the Biacore technology has dominated the commercial sector for almost two decades. Recently, other manufacturers have innovated on the concept of a flow cell and how samples are handled to increase the number of interactions that can be studied simultaneously in an automated mode, enable longer measurements, and consume less sample (Rich \& Myszka, 2007).

\subsection{Diverse biosensor configurations}

Fig. 1 depicts the way in which three different biosensor platforms address samples. The Octet is a fairly new technology that uses bio-layer interferometry by employing fiber-optic sensors incorporated on disposable tips. Another emerging technology, the ProteOn, uses surface plasmon resonance (SPR) and gold sensor chips, as does the Biacore. For more information about the principles upon which these detectors are based, the reader is referred to the manufacturers' websites; www.fortebio.com, www.bio-rad.com, and 
www.biacore.com. By reversing the configuration where now the sensors move to the samples, the Octet renders the microfluidics that delivers samples to a stationary sensor chip in the ProteOn and Biacore systems, unnecessary. The Octet adopts a well-based dip-andread format in which a column of up to eight sensor tips immerses into samples held in an open shaking microplate (Fig. 1A). Binding steps are defined by moving these sensors along the rows in the plate and the tips are discarded at the end of the assay. Dipping into samples rather than injecting them allows the Octet to measure longer association times and re-use samples within an assay or recover them for other uses. The ProteOn also processes samples in parallel, delivering them via six injections that flow perpendicularly to create a unique six-by-six interaction array (Fig. 1B). This generates 36 "reaction spots" (shown by the red squares in Fig. 1B) where flowpaths intersect and 42 "interspots" (in each direction), which provide a local-referencing option as an alternative to traditional whole-channel referencing. In contrast, the Biacore 3000 injects one sample at a time over up to four serially-addressed flow cells, one of which typically serves as a reference channel (Fig. 1C).
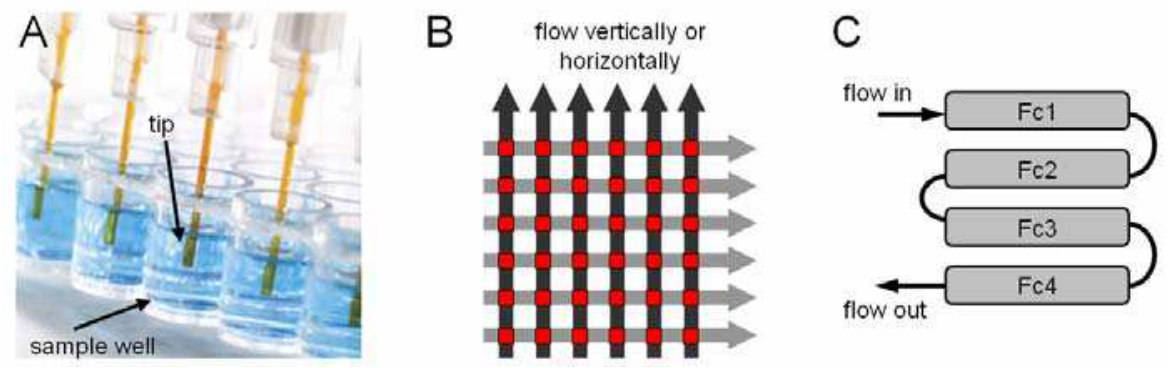

Fig. 1. Sample addressing in (A) Octet QK, (B) ProteOn XPR36, and (C) Biacore 3000 biosensors. The sample plate used in A is black.

The above-named SPR platforms support a limited number of immobilized ligands per experiment and rely upon being able to regenerate them. In contrast, the Octet can analyze up to 96 ligands per experiment on inexpensive single-use sensors that can be regenerated to make an assay more cost-effective. The throughput offered by the ProteOn may make regeneration unnecessary if the 36 interactions that can be addressed in a single binding cycle cover all the desired permutations. An unique advantage of the Octet's configuration is that ligands can be loaded offline onto batches of sensors, which increases the number of ligands that can be studied in a single run and speeds up the assay because fewer steps need to be performed online.

\subsection{The importance of benchmark studies}

It is important to assess the performances of emerging technologies relative to well-establish ones since biosensors are now routinely multiplexed to meet the demands of drug discovery for higher throughput. Determining whether there is consensus across different biosensor platforms operated by independent users has been a theme of several benchmark studies (Rich et al., 2009; Navratilova et al., 2007; Katsamba et al., 2006; Papalia et al., 2006; Cannon et al., 2004; Myszka et al., 2003). The largest and most recent of these engaged 150 participants from 20 countries who used instruments from ten different manufacturers. These collaborations enhance the biosensor community's ability to design experiments and provide insights into the variability of biosensor data where it can become difficult to 
discriminate between sub-optimal instrument quality and an unskilled user. Inspired by these studies and the continuing evolution of biosensors, we compared the performances of two parallel-processing platforms, namely the Octet QK and the ProteOn XPR36 interaction array, head-to-head with the serial flow Biacore 3000 unit that represents the current "workhorse" of the biosensor research community as judged from it being the most frequently cited platform in the literature (Rich \& Myszka, 2008). First, we addressed binding kinetics which is perceived as the signature role of biosensors based upon the abundance of literature on the topic. Then we explored competitive binding which, despite its relevance to drug discovery, is scarcely reported on in the literature.

\section{Comparing binding kinetics from different biosensors}

\subsection{Model interaction system}

For the purpose of comparing instruments side by side and to enable other investigators to reproduce our work, we adopted a commercially available antigen/antibody pair as a model system. The murine monoclonal antibody 4901 (Wong et al., 1993) was chosen because it binds various forms of calcitonin gene-related peptide (CGRP) with affinities that fall within a measurable range (high picomolar to mid-nanomolar) and allows for facile regeneration in all assay orientations. CGRP is implicated in migraine and other types of pain and thus interfering with its biological activity is relevant to drug discovery (Geppetti et al., 2005). We studied wild-type (1-37) rat-CGRP-alpha (rCGRPa) and 1-37, 26-37, and 3237 forms of human-CGRPa (hCGRPa), which spanned molecular masses of 609Da to $3806 \mathrm{Da}$ and bound antibody 4901 with affinities ranging from $0.5 \mathrm{nM}$ to about 500nM. We oriented the assay in three different ways. First, we presented naked peptides to antibodycoupled sensors. Secondly we presented Fab fragments to N-biotinylated peptides that were captured via streptavidin sensors. Lastly, we deduced an affinity of the CGRP/4901 interactions indirectly via solution competition. The following sections describe each assay orientation as published elsewhere (Abdiche et al., 2008).

\subsection{Binding solution antigen to sensors coupled with antibody}

When the goal is to generate binding kinetics, it is prudent to present a monomeric partner in solution so that its binding can be modeled easily without interference of avidity (Myszka, 1999). Therefore, one way to study the CGRP/4901 interaction was to allow the solution peptides to bind amine-coupled antibody. None of the peptides could be detected directly on the Octet due to their binding signals being within instrument noise, but all were clearly visible on the SPR platforms; small molecule analysis is routine on the Biacore 3000 (Navratilova et al., 2007; Papalia et al., 2006; Cannon et al., 2004; Myszka et al., 2003) and was first described on the ProteOn by Bravman et al., 2006. These authors introduced a method called "one-shot kinetics" that exploits the parallel injection mode of the ProteOn to deliver in a single step a six-membered dilution series of the analyte over six surfaces of immobilized ligand with varying binding capacities. This generates a robust data set because a large number of binding curves are fit simultaneously to converge upon an unique pair of kinetic rate constants, whose ratio gives a global affinity for the interaction. Using this method, we analyzed four peptides binding to multiple levels of coupled 4901 by regenerating the immobilized antibody after each "one-shot" series and duplicating every injection. The kinetic analysis of one of these peptides, namely hCGRPa 32-37, is shown in Fig. 2A. This interaction was also amenable to an equilibrium analysis (Frostell-Karlsson et 
al., 2000) as a non-kinetic route to the affinity owing to all the binding responses reaching plateau values within the association phase (Fig. 2B).
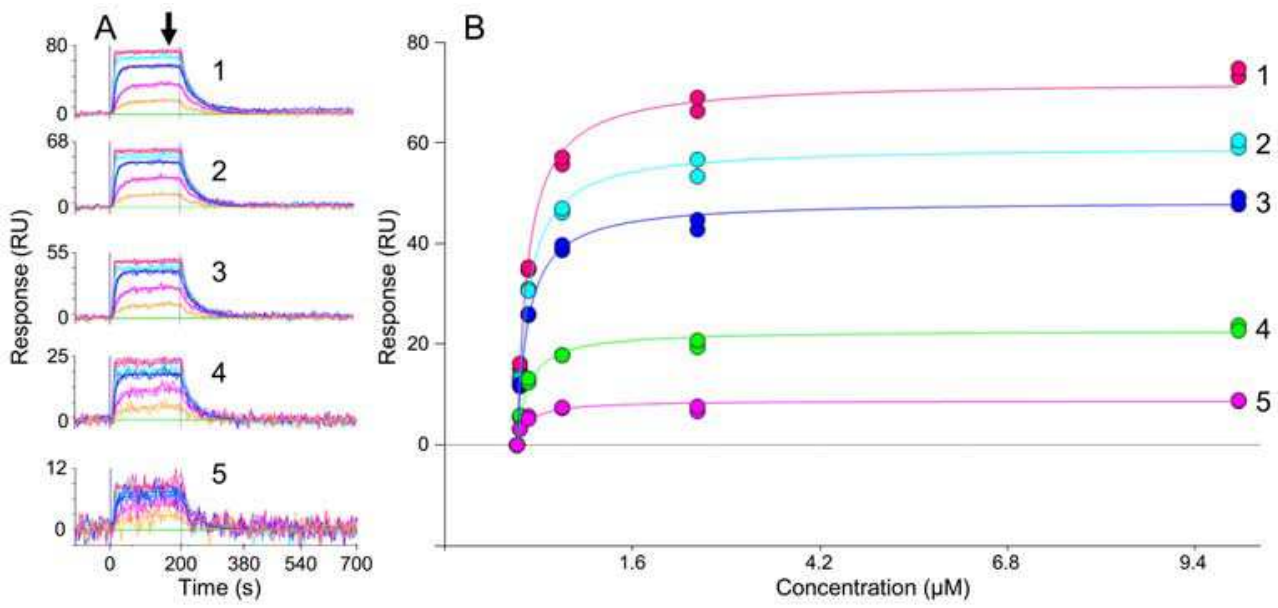

Fig. 2. One-shot kinetics on the ProteOn. (A) Primary data (noisy lines) for hCGRPa 32-37 binding five levels of coupled antibody 4901 (where 1-5 depict the high to low surface capacities) and their simultaneous fit to a simple kinetic model (solid lines) that gave a $K_{\mathrm{D}}$ of $147 \mathrm{nM} \pm 1 \%$ standard error for the fit. (B) Alternate analysis of the same data set by plotting the equilibrium binding responses (arrowed in panel A) from all five surfaces as a function of injected peptide concentration and fitting them simultaneously to a global $K_{\mathrm{D}}$ of $149 \mathrm{nM}$ $\pm 3 \%$ standard error for the fit.
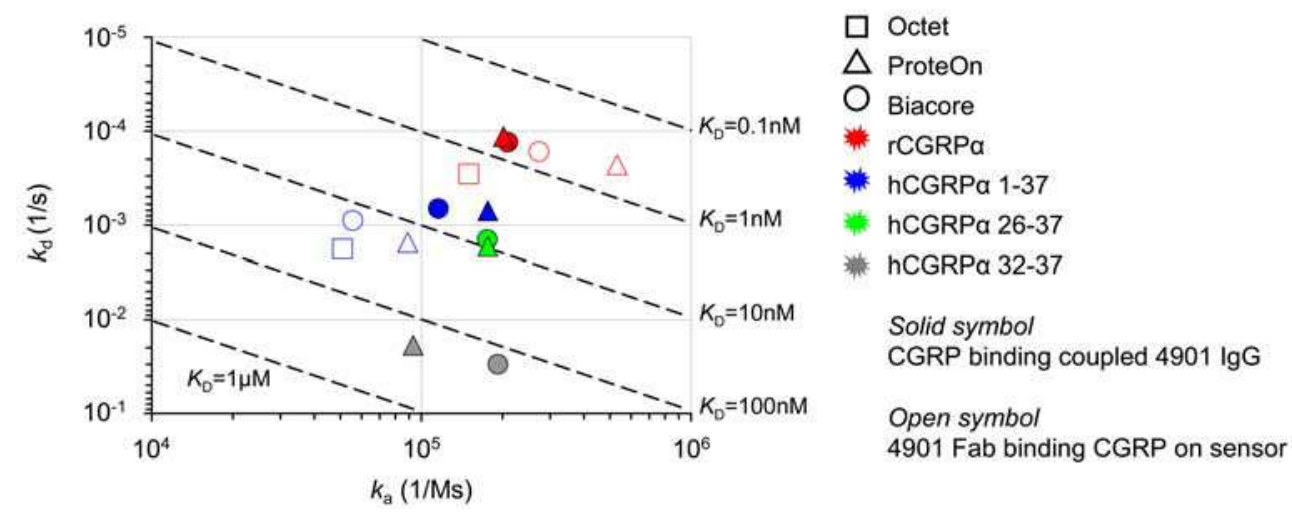

Fig. 3. Kinetic rate constants determined in two assay orientations. Not all permutations of biosensor, peptide, and assay orientation were investigated (see Table 1). Diagonal dotted lines trace the isoaffinities.

The ProteOn and Biacore platforms returned remarkably similar kinetic rate constants for an array of CGRPs binding coupled $4901 \mathrm{IgG}$ (Fig. 3, solid symbols) and the affinity ranking was consistent with 4901's known species-selectivity and its epitope that incorporates the ten most C-terminal residues of CGRP. 


\subsection{Binding solution Fab to sensors coated with peptide}

Another assay orientation that we explored was the direct binding of Fab to N-biotinylated full-length peptides on streptavidin or neutravidin sensors. This method was less convenient than the one described above because the reagents had to be modified. While the Fab was easily detectable on the Octet, it rebound the CGRP-saturated tips, to different extents depending upon the CGRP used, as evidenced by the deviation of the dissociation phase from a single exponential decay (black lines in Fig. 4A and 4B). While rebinding is a drawback of the Octet's well-based format, it was rectified by spiking the dissociation buffer with high concentrations of a tight-binding competing antigen $(100 \mu \mathrm{M}$ rCGRPa) (red lines in Fig. 4A and 4B).

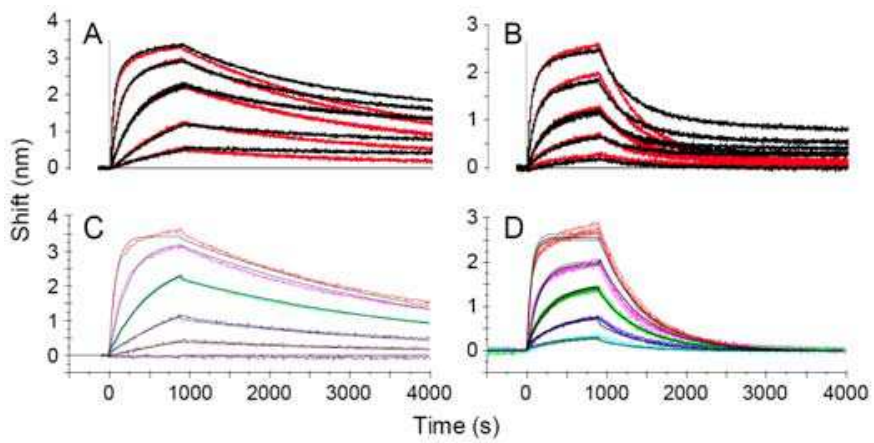

Fig. 4. Primary Octet data for "one-shot" kinetics of 4901 Fab binding streptavidin tips coated with (A) rCGRPa or (B) hCGRPa, dissociating them into buffer (black) or a sink (red). (C) and (D) show the kinetic fits (in black) of the "sinked" data (in color) in panels A and B, respectively. The global fit in D is for quadruplicate analyses on one column of tips.

Not only did the fitted kinetic rate constants and affinities (Table 1) describe the Octet data very well (Fig. 4C and 4D), but they agreed closely with those obtained for similar analyses on ProteOn and Biacore platforms (Fig. 5) and previously published values (Zeller et al., 2008). The Octet and ProteOn data were performed in a parallel "one-shot" kinetic mode whereas the Biacore data were collected in a serial multi-cycle mode by regenerating the surfaces after each injection.
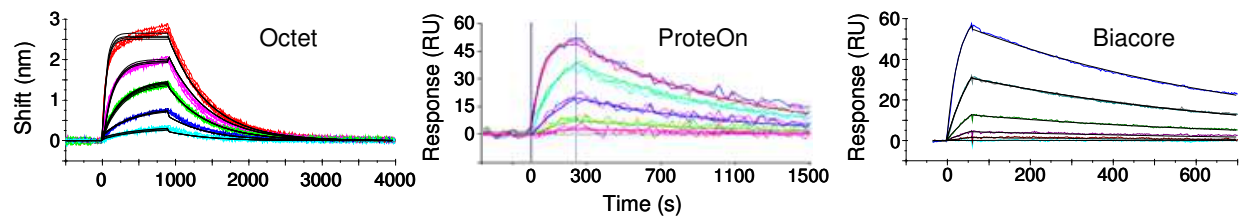

Fig. 5. Direct comparison of 4901 Fab interacting with hCGRPa surfaces on different biosensors. The measured data and their global fits are overlaid. The timing of the binding steps varied according to the biosensor used.

\subsection{Determining affinity via solution competition}

While the Octet cannot detect small molecules directly, it can access affinities indirectly via solution competition. An advantage of deducing a solution affinity is that it is unbiased by the 
assay orientation and unaffected by the immobilization process. In this type of experiment, two binding partners are mixed in solution at various concentrations and the free concentration of one of them is probed by an immobilized molecule whose affinity is not being measured. An appealing feature of our model system was that the sensors could be coated with the tight-binding rCGRPa to probe the weaker affinities of the other peptides (Fig. 6).
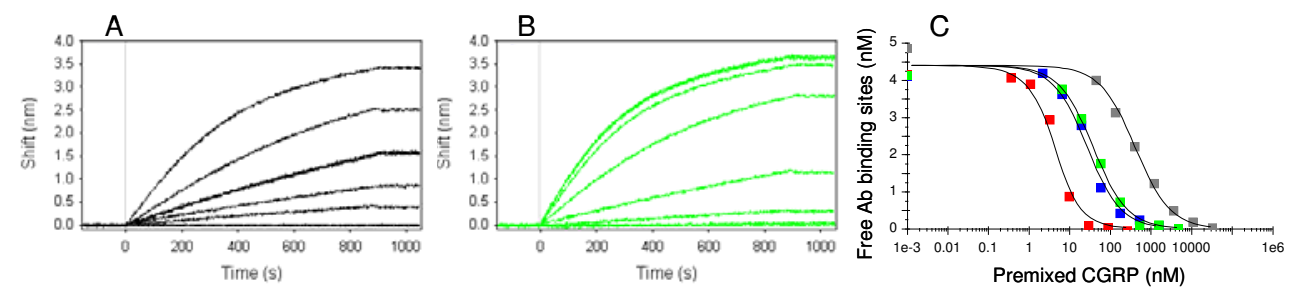

Fig. 6. Solution competition on the Octet using rCGRPa-saturated tips to probe for free antibody binding sites in (A) standard curve (twofold serial dilution of 4901) and (B) inhibition curve (fivefold serial dilution of hCGRPa 26-37 into a fixed concentration of 4901). (C) Overlay plot of the inhibition by rCGRPa (red) and 1-37 (blue), 26-37 (green), and 32-37 (grey) hCGRPa with fits in black.

Similar results were obtained on all three biosensors (Table 1), but only the Biacore was sensitive enough to work at the sub-nanomolar concentrations of fixed antibody binding sites needed to estimate the tight affinity of the rat peptide. Ideally, the concentration of the partner being detected at the sensor (in this case, the antibody) should be fixed (at or) far below the anticipated affinity of the solution interaction being measured in order for binding to be driven by affinity. If it is fixed far above the anticipated affinity of the solution interaction, then binding will be driven mainly by concentration and thus will instead determine the antibody's "active concentration" by titrating out CGRP in a stoichiometric manner.

\subsection{Comparing assay orientations in affinity measurements}

The ProteOn and Biacore returned virtually identical kinetics (Fig. 3) regardless of the assay orientation used, while those determined on the Octet were typically within twofold of them with direct binding of small molecules beyond its detection limit (Table 1). The affinity of the rCGRPa/ 4901 interaction was consistently around $0.5 \mathrm{nM}$ by SPR by all three methods, suggesting that neither binding partner had been adversely affected upon immobilization or modification. In contrast, the wild-type hCGRPa discriminated between the Fab and intact

\begin{tabular}{|l|ccc|ccc|ccc|}
\hline CGRPa & \multicolumn{6}{|c|}{ CGRP binding coupled 4901 } & \multicolumn{4}{c|}{ 4901 Fab binding CGRP on sensor } & \multicolumn{3}{|c|}{ Solution competition with IgG (or Fab) } \\
\cline { 2 - 9 } & Octet & ProteOn & Biacore & Octet & ProteOn & Biacore & Octet & ProteOn & Biacore \\
\hline Rat 1-37 & ND & 0.565 & 0.569 & 2 & 0.612 & 0.432 & ND & ND & $0.54(0.45)$ \\
Hu 1-37 & ND & 5.78 & 4 & 36 & 16.2 & 17.3 & $28(22)$ & $7(20.3)$ & $5.8(18.2)$ \\
Hu 26-37 & ND & 8.13 & 9.55 & NT & NT & NT & $38(27)$ & $34(23.6)$ & $44(23.1)$ \\
Hu 32-37 & ND & 147 & 202 & NT & NT & NT & $500(280)$ & $370(224)$ & $460(239)$ \\
\hline
\end{tabular}

Table 1. Affinities $\left(K_{\mathrm{D}}, \mathrm{nM}\right)$ of CGRPa/ 4901 interactions determined in various assay orientations on different biosensors. ND=not determined due to lack of sensitivity. $\mathrm{NT}=$ not tested. Values are the mean of replicate experiments (with typical standard deviation $<20 \%$ ) or the global fit of a single measurement (with typical standard error $<1 \%$ ) 
IgG; the Fab bound hCGRPa on chip with a threefold weaker affinity than its IgG counterpart with the trend being corroborated by solution competition measurements. In a separate experiment, we confirmed that the naked and N-biotinylated peptides bound with identical kinetics when flowed over coupled IgG. Our results demonstrate that the assay orientation can affect the results as much or more than the biosensor used.

\section{Exploring blocking assays on biosensors}

The next application we explored was competitive binding because of its relevance to drug discovery. Most drugs functioning as antagonists are aimed at blocking a natural interaction such as that between a ligand and its receptor. Monoclonal antibodies (Abs) are becoming increasingly utilized as therapeutic entities in blocking these ligand-receptor interactions. An epitope is defined as the three-dimensional region on an antigen (Ag) where an antibody binds. Discriminating Abs based on their epitope rather than their apparent affinity for the target Ag is often more important because of established protocols for affinity maturation by protein engineering, while changing an epitope to a desired one is not typically possible. We decided to illustrate the use of biosensor blocking assays in the context of epitope binning (Abdiche et al., 2009).

\subsection{Sandwiching assays in the context of epitope binning}

Biosensors are essentially mass-based detectors. Therefore, determining whether an $\mathrm{Ab}$ blocks another's ability to bind Ag is simply a yes/no read-out, regardless of the assay orientation used. Under conditions that favor complete blocking, i.e. overlapping epitopes, no binding response will be detected at the sensor for any of the arrowed interactions in Fig. 7. In contrast, a binding signal at each of these interactions identifies a pair of Abs that can coexist on the Ag at distinct, non-overlapping epitopes and thus form a sandwich complex. Using this yes/no detection of the arrowed interactions, we assign Abs to epitope bins according to their blocking profiles relative to one another.
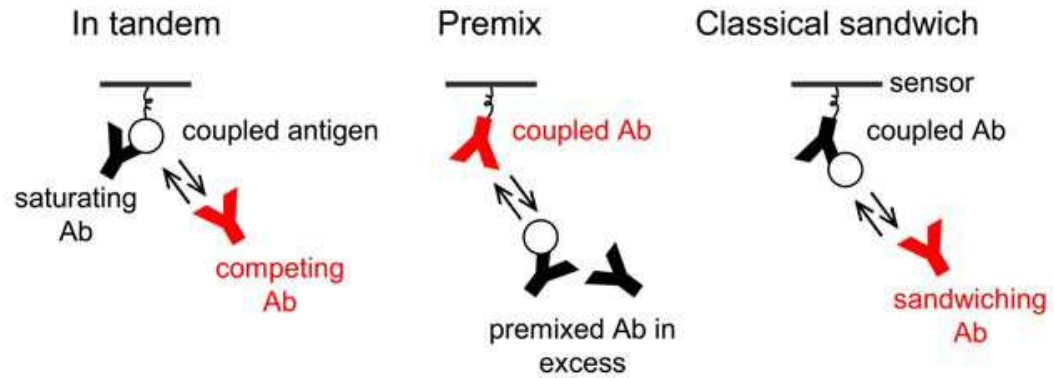

Fig. 7. Three assay orientations that can be used in epitope binning. In each case, the antigen binds the black $\mathrm{Ab}$ before the red $\mathrm{Ab}$.

In order for two Abs to belong to the same bin, they must block one another and exhibit similar blocking profiles when each is paired with the other Abs in the test panel. Thus, the observation that two Abs block one another is necessary but not sufficient to conclude that they belong to the same bin. Once two Abs have been assigned to the same bin it is possible that another $\mathrm{Ab}$ will be discovered in the future that blocks one and not the other, thus 
resolving them into different bins. We used six Abs (1-6) against a dimeric Ag to illustrate "in tandem" and "premix" methods and six different Abs (A-F) against an unrelated monomeric Ag to illustrate the "classical sandwich" method. We performed each assay on the three above-mentioned biosensors and since all biosensors gave similar results we only show data from the ProteOn because its two-dimensional interaction array makes it particularly well-suited to pairwise and combinatorial approaches to binning. The following sections describe each assay orientation in turn.

\subsection{In tandem binning}

In tandem blocking is probably the simplest conceptually. It involves coating the sensors with $\mathrm{Ag}$ and presenting two Abs one after another, namely a "saturating $\mathrm{Ab}$ " followed by a "competing $\mathrm{Ab}^{\prime}$ (Fig. 7A). Fig. 8 shows how we performed an in tandem binning on the ProteOn and addressed 36 pairs of Abs in a single binding cycle with a complete set of six "self-blocking" controls along the diagonal of the interaction array. Consolidating the results into a matrix that can be read horizontally or vertically (Table 2) revealed three blocking patterns or "bins", namely Ab1 and 3; Ab2 and 4; and Ab5 and 6. Thus, Ab1 and 3 each sandwiched with Ab5 and 6, whereas $\mathrm{Ab} 2$ and 4 blocked all six.
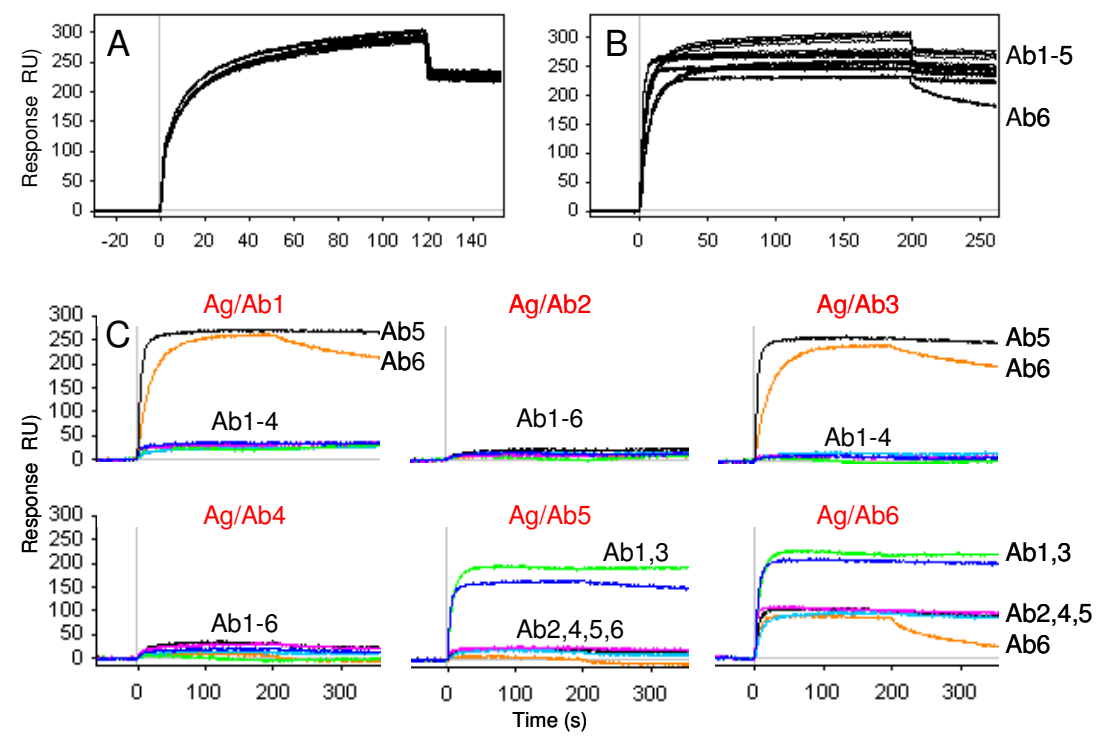

Fig. 8. In tandem binning. (A) Immobilizing Ag on all vertical channels. (B) Saturating with six Abs (1-6) on separate vertical channels. (C) Competing with the same Abs (1-green, 2-pink, 3blue, 4-cyan, 5-black, and 6-orange) in the horizontal direction, where the plots are grouped and named (in red) by the "saturating" Ab that was prebound to the immobilized Ag.

\subsection{Premix binning}

We used the same set of reagents, namely Abs1-6 raised against a dimeric Ag, to illustrate the premix approach, which involved premixing the Ag separately with a large molar excess of each $\mathrm{Ab}$, and then presenting these preformed complexes to an array of Abs coupled to 


\begin{tabular}{|c|cccccc|}
\hline Saturating Ab & \multicolumn{7}{|c|}{ Competing Ab } \\
& 1 & 2 & 3 & 4 & 5 & 6 \\
\hline 1 & $\mathrm{SB}$ & $\mathrm{B}$ & $\mathrm{B}$ & $\mathrm{B}$ & $\mathrm{NB}$ & $\mathrm{NB}$ \\
2 & $\mathrm{~B}$ & $\mathrm{SB}$ & $\mathrm{B}$ & $\mathrm{B}$ & $\mathrm{B}$ & $\mathrm{B}$ \\
3 & $\mathrm{~B}$ & $\mathrm{~B}$ & $\mathrm{SB}$ & $\mathrm{B}$ & $\mathrm{NB}$ & $\mathrm{NB}$ \\
4 & $\mathrm{~B}$ & $\mathrm{~B}$ & $\mathrm{~B}$ & $\mathrm{SB}$ & $\mathrm{B}$ & $\mathrm{B}$ \\
5 & $\mathrm{NB}$ & $\mathrm{B}$ & $\mathrm{NB}$ & $\mathrm{B}$ & $\mathrm{SB}$ & $\mathrm{B}$ \\
6 & $\mathrm{NB}$ & $?$ & $\mathrm{NB}$ & $?$ & $?$ & $\mathrm{SB}(?)$ \\
\hline
\end{tabular}

Table 2. Blocking matrix for in tandem binning. $\mathrm{Y}=$ blocks, $\mathrm{N}=$ does not block, SS=selfsandwich control, ? = intermediate.

the chip (Fig. 7B). Three controls are needed to validate the assay. First, the premixed Abs themselves in the absence of Ag should be presented to the naked coupled Abs to confirm that they do not bind one another non-specifically; indeed, one should confirm empirically that all three assay orientations depicted in Fig. 7 are free of non-specific $\mathrm{Ab} / \mathrm{Ab}$ interactions. Second, the Ag is premixed with buffer to establish the Ag-binding response expected to each of the coupled Abs. Third, the Ag should be premixed with a solution counterpart of each coupled $\mathrm{Ab}$ on the chip to demonstrate that the premixed Abs are at high enough concentrations to self-block.

On the ProteOn, a premix binning experiment involves coupling an array of Abs along the vertical channels and then injecting Ag premixed with an array of Abs along the horizontal channels. Ag premixed with buffer is used as the sixth sample. Thus, in a single binding cycle, we addressed 36 interactions that incorporated six Ag-only controls, five self-blocking controls, and 25 sandwiching pairs. Relative to the Ag-only response, a premixed Ab either abolished or augmented binding, consistent with it respectively blocking or sandwiching with the coupled Ab (Fig. 9). Of the six Abs tested, only Ab6 (orange curve) did not fully block Ag binding at the concentration tested, even against itself when coupled on chip, consistent with this Ab's weak affinity for the Ag. The premix binnings agreed with those determined by tandem binning.
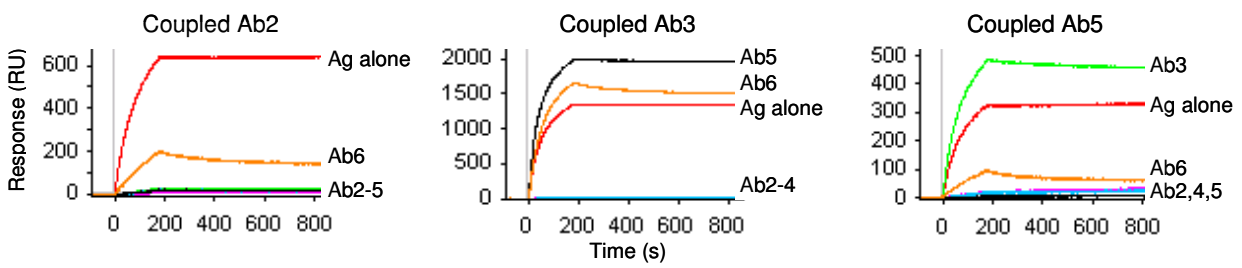

Fig. 9. Premix binning. One example of each blocking pattern is shown, grouped by coupled Ab. Premixed Abs are distinguished by color (2-pink, 3-green, 4-blue, 5-black, 6-orange, and none-red). The different response values of the three panels reflect the different coupled levels of $\mathrm{Abs} 2,3$, and 5 .

\subsection{Classical sandwich binning}

The classical sandwich assay involves coupling an $\mathrm{Ab}$ onto the sensor, binding $\mathrm{Ag}$, and then binding another $\mathrm{Ab}$ (Fig. 7C). Only monomeric Ags can be binned in this way because multivalent Ags could potentially bind the coupled $\mathrm{Ab}$ with one subunit and the solution 
$\mathrm{Ab}$ with another subunit, even if the two Abs in question bound overlapping epitopes. On the ProteOn, this assay involves coupling six Abs in the vertical direction and then rotating to the horizontal direction to inject Ag followed by an array of Abs. By matching the sandwiching Abs to the coupled ones, a complete set of "self-sandwich" controls are generated along the diagonal of the interaction array. Based on the patterns of blocking activity shown for the six Abs in Fig. 10 when paired against one another, B and F binned together, $\mathrm{D}$ and $\mathrm{E}$ binned together, and $\mathrm{A}$ and $\mathrm{C}$ were unique because they each sandwiched with the other five Abs.
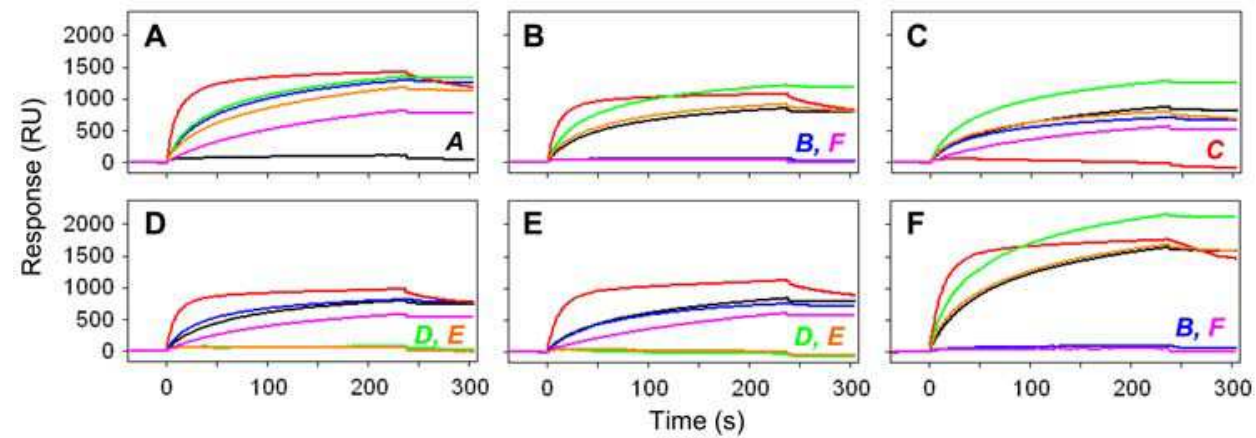

Fig. 10. Classical sandwich binning. Panels are grouped and labeled by the coupled Ab (A-F) and colored by the sandwiching $\mathrm{Ab}$ (A-black, B-blue, C-red, D-green, E-orange, and F-pink).

\subsection{Intermediate responses}

Although it is obvious in most of our examples when Abs did and did not bind, some Abs bound with intermediate responses that introduced uncertainty into whether they blocked or sandwiched. For example, the panel marked Ag/ Ab6 in Fig. 8C shows an in tandem binning result where the competing array was exposed to "free" and "Ab-bound" Ag because Ab6 dissociated rapidly from the Ag (arrowed in Fig. 8B) rather than "saturating" it, as desired. However, when Ab6 was used in the "competing" step (orange curves in Fig. 8C) it clearly binned with Ab5 (black curves). Similarly, premixing Ab6 gave an intermediate binding response (orange curves, Fig. 9) because it was at a concentration below its affinity for the $\mathrm{Ag}$ (which was unknown at that time). In general, weak binders like Ab6 should be assigned the role of "competing" Ab when studied in tandem or "on chip" in the premix or classical sandwich methods. However, Abs that dissociate rapidly from their Ag may be inappropriate candidates for coupling in classical sandwich assays because there may be insufficient $\mathrm{Ag}$ bound when a sandwiching $\mathrm{Ab}$ is presented subsequently. Since weak binders can often give ambiguous results when studied in some assay orientations, it is prudent to switch the roles of the two Abs in question and/or explore other orientations to verify a result.

$\mathrm{Ag}$ heterogeneity is another possible source of ambiguity in interpreting biosensor blocking data because consensus across all three assay orientations assumes that the Ag is a uniform population. If it is not, the assay orientations may give conflicting outcomes.

\section{Future directions}

Recently, a number of next-generation Octet units have been released that provide solutions to some of the shortcomings of the QK platform. One improvement is a better signal-to- 
noise that may now make the direct detection of small molecules possible and allow for lower surface capacities that may be preferred for kinetic runs. Moreover, their 384-well plate formats enable higher throughputs by addressing 16 interactions at once and trays of sensor tips can be re-racked to extend their lifetime.

\section{Conclusion}

Assay orientation can affect the results as much or more than the biosensor used. While the ProteOn XPR36 and Biacore 3000 platforms returned virtually identical affinities within each assay orientation tested, the Octet $\mathrm{QK}$ 's lower sensitivity made it amenable to fewer uses and required a sink to abolish rebinding artifacts. Despite these limitations, the Octet's affinities were within twofold of those determined by SPR. Binning results were comparable across the three biosensors, but differed in throughput due to each platform's unique configuration that could be exploited in creative assay design. Biosensors are versatile and reliable tools that continue to evolve and provide valuable information on diverse molecules in a research, diagnostic, and drug discovery setting above and beyond binding kinetics.

\section{Acknowledgement}

I gratefully acknowledge Arvind Rajpal and Pavel Strop for their useful comments.

\section{References}

Abdiche, Y., Malashock, D., Pinkerton, A., Pons, J. (2008). Determining kinetics and affinities of protein interactions using a parallel real-time label-free biosensor, the Octet. Anal. Biochem., 377., 2., (Jun) 209-217.

Abdiche, Y., Malashock, D., Pinkerton, A., Pons, J. (2009). Exploring blocking assays using Octet, ProteOn, and Biacore biosensors. Anal. Biochem., 386., 2., (Mar) 172-180.

Bravman, T., Bronner, V., Lavie, K., Notcovich, A., Papalia, G., Myszka, D. (2006). Exploring "one-shot" kinetics and small molecule analysis using the ProteOn XPR36 array biosensor. Anal. Biochem., 358., 281-288.

Cannon, M. et al. (2004). Comparative analyses of a small molecule/enzyme interaction by multiple users of Biacore technology. Anal. Biochem., 330., 1., (Jul) 98-113.

Frostell-Karlsson, A., Remaeus, A., Roos, H., Andersson, K., Borg, P., Hämäläinen, M., Karlsson, R. (2000). Biosensor analysis of the interaction between immobilized human serum albumin and drug compounds for prediction of human serum albumin binding levels. J. Med. Chem. 43., 10., (May) 1986-1992.

Geppetti, P. Capone, J., Trevisani, M., Nicoletti, P., Zagli, G., Tola, M. (2005). CGRP and migraine: neurogenic inflammation revisited. J. Headache. Pain., 6., 2., (Apr) 61-70.

Katsamba., P. et al. (2006). Kinetic analysis of a high-affinity antibody/antigen interaction performed by multiple Biacore users. Anal. Biochem., 352., 208-221.

Myszka, D. (1999). Improving biosensor analysis. J. Mol. Recognit., 12., 279-284.

Myszka, D. et al. (2003). The ABRF-MIRG'02 study: assembly state, thermodynamic, and kinetic analysis of an enzyme/inhibitor interaction. J. Biomol. Tech., 14., 4., (Dec) 247-269.

Navratilova, I. et al. (2007). Thermodynamic benchmark study using Biacore technology. Anal. Biochem., 364., 1., (May) 67-77. 
Papalia, G. et al. (2006). Comparative analysis of 10 small molecules binding to carbonic anhydrase II by different investigators using Biacore technology. Anal. Biochem., 359., 1., (Dec) 94-105.

Rich, R. \& Myszka, D. (2007). Higher-throughput, label-free, real-time molecular interaction analysis. Anal. Biochem., 361., 1-6.

Rich, R. \& Myszka, D. (2008). Survey of the year 2007 commercial optical biosensor literature. J. Mol. Recognit., 21., 6., (Nov-Dec) 355-400.

Rich, R. et al. (2009). A global benchmark study using affinity-based biosensors. Anal. Biochem., 386., 2., (Mar) 194-216.

Wong, H., Taché, Y., Lloyd, K., Yang, H., Sternini, C., Holzer, P., Walsh, J. (1993). Monoclonal antibody to rat alpha-CGRP: production, characterization, and in vivo immunoneutralization activity. Hybridoma, 12., 93-106.

Zeller, J., Poulsen, K., Sutton, J., Abdiche, Y., Collier, S., Chopra, R., Garcia, C., Pons, J., Rosenthal, A., Shelton, D. (2008). CGRP function-blocking antibodies inhibit neurogenic vasodilatation without affecting heart rate or arterial blood pressure in the rat. Br. J. Pharmacol. 155., 7., (Dec) 1093-1103. 


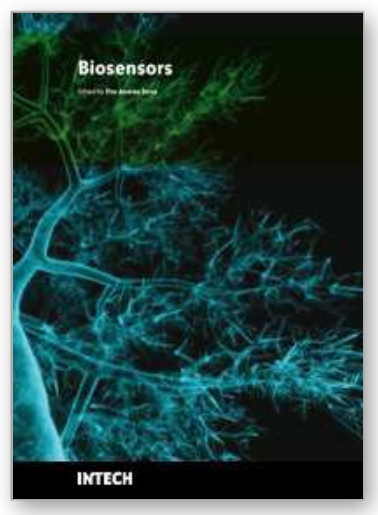

\author{
Biosensors \\ Edited by Pier Andrea Serra
}

ISBN 978-953-7619-99-2

Hard cover, 302 pages

Publisher InTech

Published online 01, February, 2010

Published in print edition February, 2010

A biosensor is defined as a detecting device that combines a transducer with a biologically sensitive and selective component. When a specific target molecule interacts with the biological component, a signal is produced, at transducer level, proportional to the concentration of the substance. Therefore biosensors can measure compounds present in the environment, chemical processes, food and human body at low cost if compared with traditional analytical techniques. Bringing together researchers from 11 different countries, this book covers a wide range of aspects and issues related to biosensor technology, such as biosensor applications in the fields of drug discovery, diagnostics and bacteria detection, optical biosensors, biotelemetry and algorithms applied to biosensing.

\title{
How to reference
}

In order to correctly reference this scholarly work, feel free to copy and paste the following:

Yasmina Noubia Abdiche (2010). Complementary Use of Label-Free Real-Time Biosensors in Drug Discovery of Monoclonal Antibodies, Biosensors, Pier Andrea Serra (Ed.), ISBN: 978-953-7619-99-2, InTech, Available from: http://www.intechopen.com/books/biosensors/complementary-use-of-label-free-real-time-biosensors-indrug-discovery-of-monoclonal-antibodies

\section{INTECH}

open science | open minds

\section{InTech Europe}

University Campus STeP Ri Slavka Krautzeka 83/A 51000 Rijeka, Croatia Phone: +385 (51) 770447 Fax: +385 (51) 686166 www.intechopen.com

\section{InTech China}

Unit 405, Office Block, Hotel Equatorial Shanghai No.65, Yan An Road (West), Shanghai, 200040, China 中国上海市延安西路65号上海国际贵都大饭店办公楼405单元 Phone: +86-21-62489820

Fax: +86-21-62489821 
(C) 2010 The Author(s). Licensee IntechOpen. This chapter is distributed under the terms of the Creative Commons Attribution-NonCommercialShareAlike-3.0 License, which permits use, distribution and reproduction for non-commercial purposes, provided the original is properly cited and derivative works building on this content are distributed under the same license. 